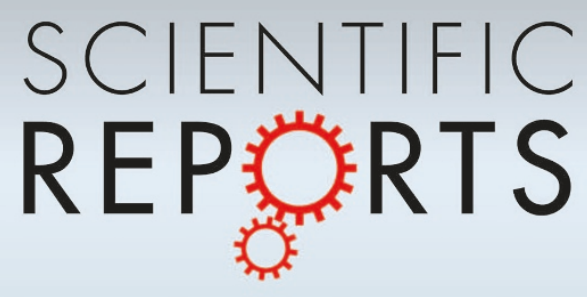

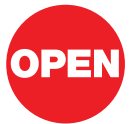

SUBJECT AREAS:

BIOINSPIRED MATERIALS

MECHANICAL ENGINEERING

BIOMIMETICS

APPLIED PHYSICS

Received

2 December 2012

Accepted

2 May 2013

Published

16 May 2013

Correspondence and requests for materials should be addressed to

Y.Q. lqinyong@|zu. edu.cn)

\section{Vibration driven vehicle inspired from grass spike}

\author{
Suo Bai ${ }^{1}$, Qi Xu' \& Yong Qin ${ }^{1,2}$
}

'Institute of Nanoscience and Nanotechnology, Lanzhou University, Lanzhou 730000, China, ${ }^{2}$ Beijing Institute of Nanoenergy and Nanosystems, Chinese Academy of Sciences, Beijing 100085, China.

Searching and detecting in some harsh environments such as collapsed buildings, pipes, small cracks are crucial for human rescue and industrial detection, military surveillance etc. However, the drawbacks of traditional moving modes of current vehicles make them difficult to perform such tasks. So developing some new vehicles is urgent. Here, we report a Setaria viridis spike's interesting behavior on a vibrating track, and inspired by that phenomena we develop a concept for cargo delivery, and give a detailed discussion about its working mechanism. This vehicle can move on a wide range of smooth and rough surfaces. Moreover, its climbing capability in tilted and even vertical smooth pipe is also outstanding. These features make it suitable for search-rescue, military reconnaissance, etc. Finally, this vehicle can be reduced into micro/ nano-scale, which makes it would play an important role in target-drug delivery, micro-electromechanical systems (MEMS).

T raditional vehicles such as cars, motorcycles, have been widely used to transport passengers and cargoes. In normal environments, these vehicles can work very well. However, in some harsh environments such as the collapsed buildings left by natural and human-induced disasters, small cracks, pipes, the traditional vehicles couldn't work properly. As for the collapsed buildings, there exist too many extrusive objects, twisted steels and some other obstacles, which make the traditional vehicles fail to propel themselves through. Another great challenge is that this kind of environment is very unstable; the perturbation incurred by the traditional vehicles would destroy the environment which will make some tasks such as rescue and searching impossible. The drawbacks of the traditional vehicles are also manifested in the areas such as small cracks, pipes. In these areas, the confined space makes the traditional vehicles fail to work. However, these environments are often faced in the industrial inspection and so on. So developing effective vehicles which can work properly in these environments is greatly demanded. At the same time, the rapid development of micro/nanotechnology needs the moving robots in the same size scale. An arising solution is based on mobile robots. In 2001, the first mobile robot was used for searching and rescue during the 911 event $^{1,2}$. The conventional robots usually convert the mechanical energy produced by the motors and engines to work via wheels, tracks and so on ${ }^{3-6}$. These robots are expected to work on smooth surfaces with small roughness. However, the practical environments are much more complex than that. In order to reconcile the robots with the harsh environments, some of wheel-less robots have been developed such as wheel-less serpentine robot $^{7,8}$, wall climbing robot ${ }^{9,10}$ and crawling toy "hexbug ${ }^{111}$. However, these kinds of robots are composed of many moduli, which increases their complexities in fabricating and motion coordinating, and makes it almost impossible to scale down them to micro/nano-scale to meet some vital demands in the fields such as MEMS, targeted drug delivery, in-vivo thrombus removal. So developing some simple, scalable, efficient wheel-less vehicles which can work properly ranging from macroscopic to microscopic areas and giving a detailed description about its working mechanism is needed.

The generous nature will never be too stingy to supply people with various kinds of inspirations, such as the lotus leaf's self-cleaning property via a hierarchical double structure ${ }^{12,13}$, moth eyes' high light transmission property via a nanostructuctured film composed of a hexagonal pattern of bumps ${ }^{14}$, water strider's walking ability on the pond's surface via a superhydrophobic coating realized by a the leg's large number of oriented tiny hairs with fine nanogrooves ${ }^{15}$, gecko foot's high adhesive force due to the foot's spatulae tipped setae ${ }^{16,17}$. Inspired by these structures, many bionic-based devices with practical or potential applications have been made such as self-cleaning surface ${ }^{18}$, anti-reflection coatings ${ }^{19,20}$, artificial dry adhesives ${ }^{21,22}$. Here, we study the Setaria viridis spike's moving behavior on vibrating cotton wire and this is the first study of this interesting behavior. After analyzing its working mechanism, we fabricate a vibration driven vehicle (VDV) using a polydimethylsiloxane (PDMS) tube with slant pillars around it according to the character of the spike. This VDV possesses the most key factors of the spike. Moreover, it can utilize the vibrating energy more effectively to make a directional 
motion. In addition, it can work on a rather smooth surface such as glass tube, plastic tube. Its motion can be steered by a pre-set orbit, which supplies us with one easy way to control the VDV's route. These features make the VDV outstanding in handling situations such as searching survivors under building ruins incurred by natural disasters and cargo delivery in some severe environment. Moreover, it is technically feasible to reduce the size of the VDV into micro/ nano-scale, so it possesses potential applications in nanotechnology such as MEMS and biomedical area.

\section{Results}

In some regions of China, people enjoy themselves by contesting with each other using a pair of grass spikes (Supplementary Movie S1). Fasten a cotton wire around two sticks horizontally to form a track with proper tensions. Then each player chooses a suitable spike for himself, according to some criteria summarized from experiences such as the spike's weight, straightness. Next, the competitors place the spikes face to face on the track. The players stimulate the track to vibrate by rubbing the sticks with stones, at the same time, the spikes mounted on the track begin to move against each other, the competitor whose spike pushes that of the other's out of the track would win this game.

In order to examine the spike's behavior in detail, we place only one spike on the track. The experimental result shows that the spike can move continuously under the vibration, moreover its moving direction depends on the style of how you place it on the track. When the style of the spike's placement changes from parallel to antiparallel with the track, its moving direction will also change correspondingly (Fig. 1a, Supplementary Movie S2). According to this spike's characteristics, it is natural to think that the spike may serve as a prototype of a new kind of vehicle. In order to make that viewpoint more convincible, we test the spike's ability for cargo delivery. The grass spike loaded with a weight via a pin is put on a tilt track, and it can move upward when vibrating the track (Fig. 1c, Supplementary Movie S3). From these experimental results, we hypothesize it is the structure along with the material's elastic property that determines the spike's moving behavior. To further confirm this, we place a piece of hairy rat fur whose structure is similar to the spike on a vibrating surface of a drum. The fur can make the directional motion in this case (Supplementary Movie S4), which provides a proof for our conjecture. Secondly, it is the interaction between the grass spike's awns and the track that makes this kind of motion possible. In this case, when increasing the interacting area, the spike would gain an enhanced speed. In order to verify that, we replace the above track with a cloth which is rolled up into a semi-tubular shape (Supplementary Fig. S1a) to increase the interaction area and indeed, the spike gains an enhanced motion which is 3.4 times of that on the cotton wire (Fig. S1b, Supplementary Movie S5). Finally, we encapsulate the spike into a plastic tube where the spike could get a maximal interacting area to enhance its mobility, and the spike can climb vertically (Fig. 1b, Supplementary Movie S6).

From the experimental results, we conclude that it is the structure along with the material's elastic properties that generates the spike's directional motion utilizing the vibrating energy. The detailed structure of the spike mounted on the track is shown in Fig. 2a. A further microscopic observation shows that the spike has slant awns with diameters 60-110 $\mu \mathrm{m}$ and length 6-10 $\mathrm{mm}$ around it, and the slant angle of the awn with respect to the peduncle ranges from $40^{\circ}$ to $60^{\circ}$. As shown in above experiments, the spike always moves towards one direction no matter which side of the stick is stimulated and whether the cotton wire is horizontal or tilted, so the external stimuli is not a crucial factor that determines the spike's moving direction. In order to simplify the analysis about the directional motion, we assume that the external stimuli are homogeneous. Apparently, there should be one mechanism to break the forward and backward symmetry to make the spike move toward one direction. And the only way to break this symmetry is via the spike's anisotropic structure. The spike's slant awns make the forward direction is not equivalent to the backward direction. The vibrating mode of the track is very complicated, in order to grasp its most important parts and neglect the trivial things, we decompose the vibration into two categories according to its direction with respect to the substrate, i.e., the back and forth vibrating mode as well as the up and down vibrating mode. First, we use the jagged surface as shown in Fig. $2 \mathrm{~b}$ and $\mathrm{c}$ to represent the rough track and suppose the slant angle of the sawtooth along the substrate plane is $\theta$, the slant angle between the substrate plane and the horizontal plane is $\beta$. As for the back and forth vibrating mode, in the forward period of the track's motion, due to the inertia of the awns, the spike's

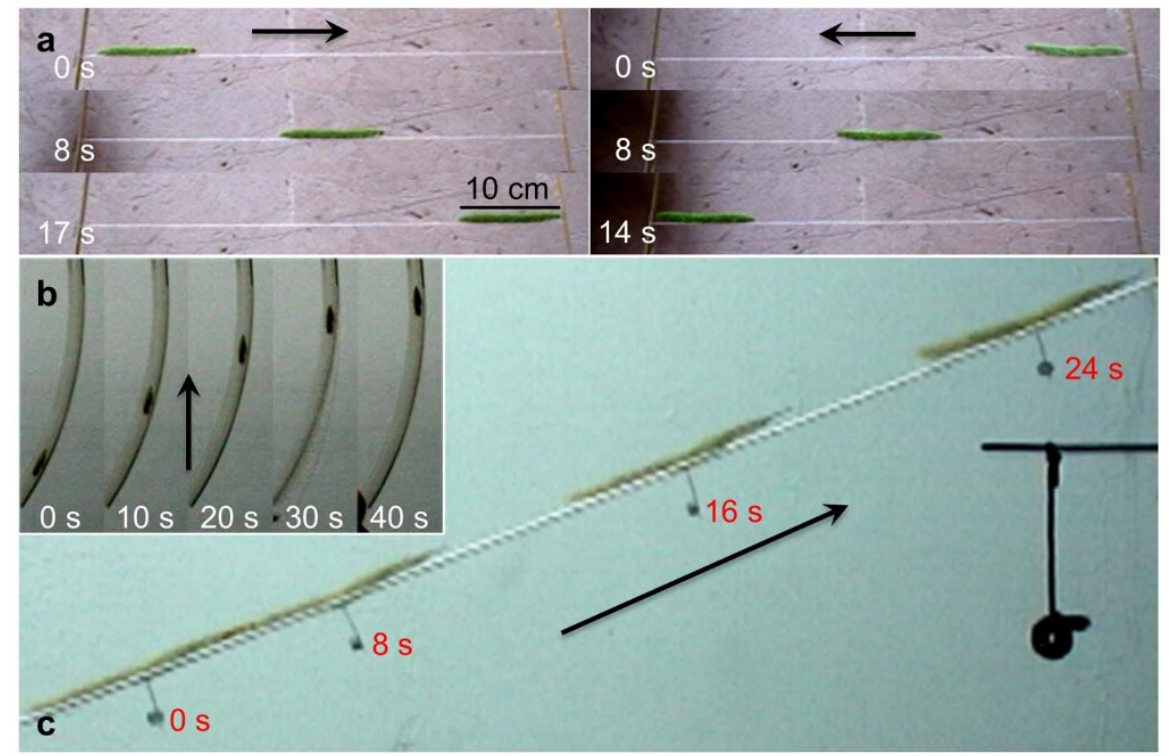

Figure $1 \mid$ Motion of the grass spike on different tracks. (a) A spike's motion on a vibrating cotton wire with the spike's head towards right (left panel) and left (right panel), respectively. (b) Images of the spike moving upward in a vertical plastic tube. (c) Overlain images of the spike loaded with a weight via a pin moving along a tilt cotton wire. The black wire on the right side indicates the plumb line. The arrows in all the figures point from the tail to the head of the spike. And they also point out the spikes' moving direction. 


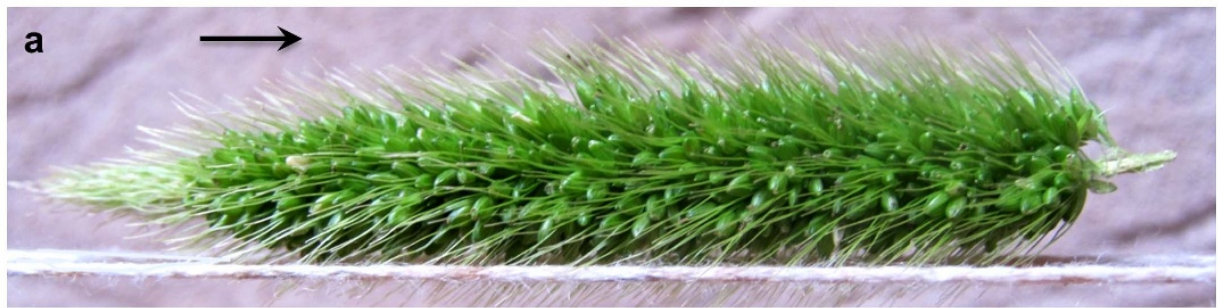

b

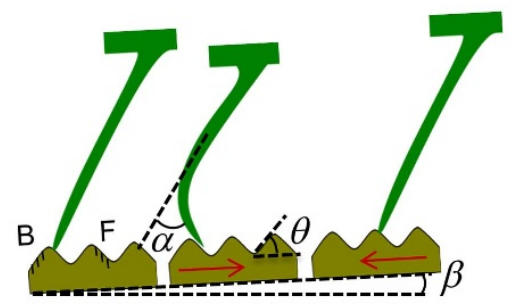

C

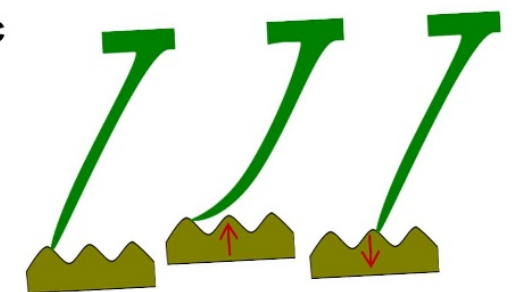

Figure $2 \mid$ Optical photograph of the spike and schematic of its working principle. (a) Side view of a spike placed on a cotton wire. The arrow points from the tail to the head of the spike. (b) The schematics of the spike in the original state, the first and second half period of the back and forth vibration. (c) The schematics of the spike in the original state, the first and second half period of up and down vibration. The arrows in (b) and (c) point out the moving direction of the track and $\alpha, \theta$ and $\beta$ are the deflection angle of the awn and the slant angle of the sawtooth and slant angle of the substrate, respectively. $\mathrm{F}$ (Forward) and B (Backward) are two kinds of surfaces in the sawtooth.

movement is not so fast to keep up with that of the track, so relative to the track its awns move backward and get stuck by the sawtooth of the jagged surface as shown in the middle panel of Fig. 2b. Suppose the awn's deflection angle is $\alpha$. The normal force $F_{N}$ between the awn and the sawtooth is equal to $G * A * \alpha$, where $G$ is the awn's shear modulus, $A$ is the contact area between the awn and the sawtooth. In the backward period of the track's motion, the bent slant awns will move forward relative to the track, and the normal force $F_{N}$ would decrease gradually to zero and we suppose the process is a linear one and happens in a time interval $t$. According to the impulse-momentum theorem, the awn will get a velocity $v_{0}$ with magnitude $G * A * \alpha * t /(2 m)$, where $m$ is the mass of the awn, and direction perpendicular to the sawtooth. After this, the awn will exhibits projectile motion. And during this process the awn will make a leap whose magnitude

$$
l=(G * A * \alpha * t / m)_{*}^{2}[\tan (\alpha+\beta)-\tan (\beta)] * \cos ^{2}(\alpha+\beta) /\left[2 * g_{*} \cos (\beta)\right],
$$

where $g$ is the acceleration of gravity. The awn will make a forward motion when it is got stuck by side F of the sawtooth, as opposed to
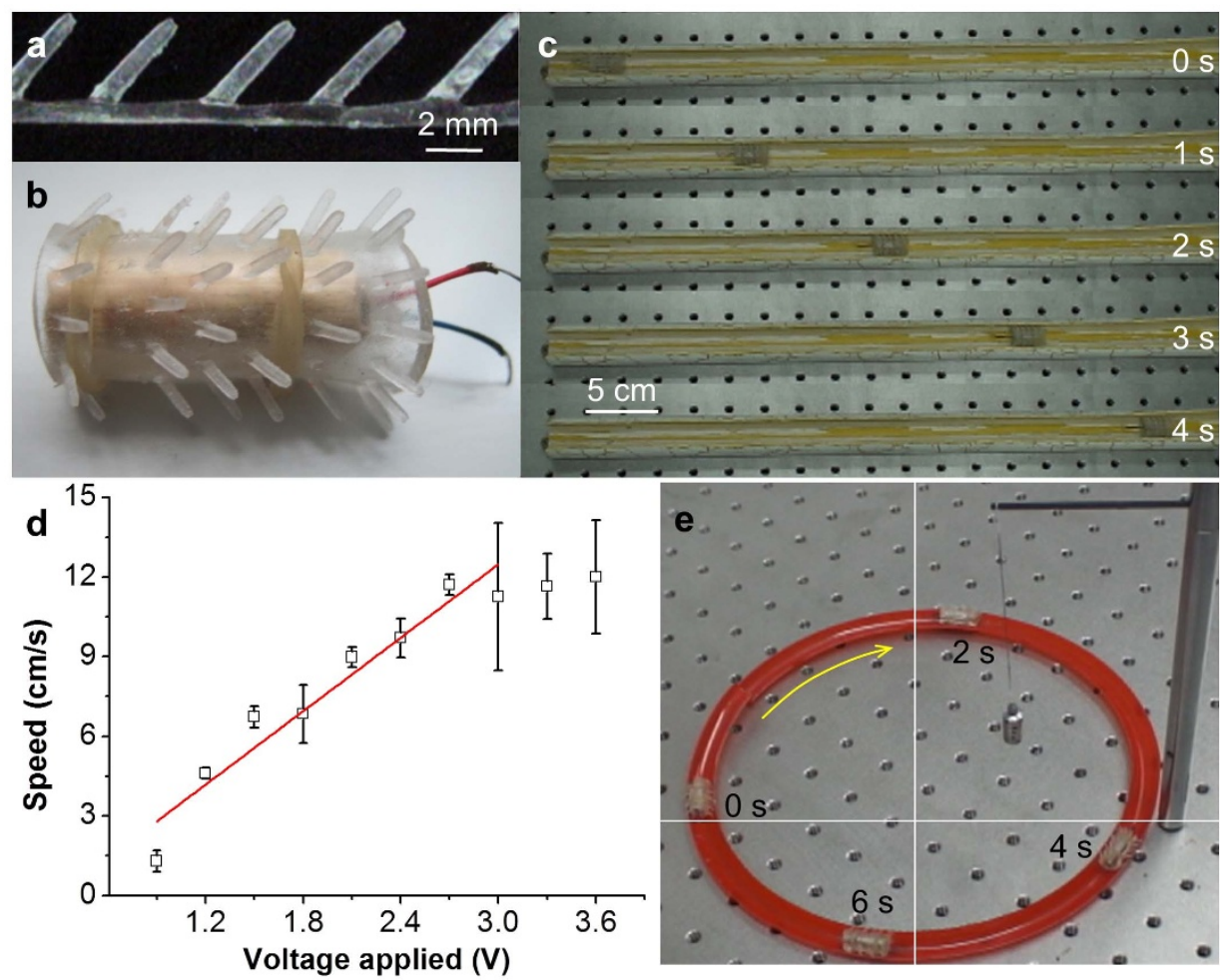

Figure 3 Optical photograph of VDV and its motion characterization. (a) Side view of PDMS pillar array. (b) Optical photograph of VDV, with a vibrating motor mounted in. (c) Images of a VDV moving on a horizontal groove. (d) The relationship between the VDV's speed and the voltage applied on the motor. Below the rated voltage $(3 \mathrm{~V})$ of the motor, a linear fit can be used to represent this relationship. Each error bar indicates the standard deviation. (e) Images of the VDV moving in a circular plastic tube. The space between the neighbor holes in (c) and (e) is $2.54 \mathrm{~cm}$. 
that the awn will make a backward motion when it is got stuck by side B. If the awn is vertical, the awn has the same probability to be got stuck by the side F and B. So it will not move on either direction and will shake randomly. When the awn tilted like the case shown in Fig. 2, the awn has a higher probability to be got stuck by side F, so the awn will make a forward motion. When the stimuli are successively applied, the spike would move forward continuously. As for the up and down vibrating mode, in the upward period of the track's motion, due to the inertia of the awns, the slant awns will get squeezed, during this process the elastic energy is stored in the bent awns as shown in the middle panel of Fig. 2c. In the downward period of the track's motion, the awns will move upward relative to the jagged surface, the squeezed slant awns will make a leap, like the case of back and forth vibrating mode, in this process the grass will move forward. And the up and down vibrating mode of the VDV can be used to explain the hexbug's movement ${ }^{11}$. From our analysis we can see the spike will make a step at each period in both cases and the slant awns are crucial in the process when the elastic energy is converted to directional motion. The motion's direction is determined by the orientation between the awns and the track. So when placing the grass spike on the track with its tail and head interchanged, the tiny step's moving direction would be altered. Though we use a jagged surface to illustrate the spike's working mechanism, the spike can also move on the smooth surfaces with a very small roughness such as plastic surface as shown in Fig. 1b. The reason is that the conventional smooth surfaces such as plastic and glass surfaces are relative smooth surfaces with the very small roughness, which means that they are macroscopic smooth but microscopic rough. And the spike can move on any surfaces as long as their roughness is comparable with the tip of the spike in size.

Though the spike has exhibited so many merits compared with the traditional vehicles, its endurance is not good and it needs work under external stimuli. Moreover, from the analysis of the spike's working mechanism, we find in order to make the spike move forward, the local vibration near the spike is sufficient. So it needn't to stimulate the whole track to vibrate. In order to utilize the vibrating energy more effectively and improve this vehicle's robustness, we use a PDMS tube with slant pillars around it as a VDV, as shown in Fig. $3 \mathrm{a}$ and b. Moreover, in order to improve its autonomy for motion control, we mount an internal vibrating motor inside the tube, with that we can control the vibrating frequency, which is crucial to the motion. The VDV can make the directional motion well, as shown in Fig. $3 c$ and Supplementary Movie S7. Moreover, the speed of the VDV increases linearly with the applied voltage when it is smaller than the motor's rated voltage $3 \mathrm{~V}$. Thus, by adjusting the voltage applied on the vibrating motor, we can control the vehicle's speed as shown in Fig. 3d, which can be used to meet different demands in practical use. Based on our previous analysis, we give a simple formula to elaborate this phenomenon, $v=f_{*} l$, where $v$ is the VDV's speed, $f$ is the vibration frequency, $l$ is the leaping distance of the VDV in one period of the back and forth/up and down mode. The speed of the VDV increases linearly with the vibration frequency, as the VDV could make more leaps per unit time at a higher frequency. Below the motor's rated voltage, the vibrating frequency nearly increases linearly with the applied voltage. So the speed of the VDV will increase linearly with the applied voltage. Given a preset track, the vehicle can move along it, as shown in Fig. $3 \mathrm{e}$ and Supplementary Movie S8, which demonstrates a simple way for motion controlling. Next, we place the VDV into a glass tube, it can climb up vertically (Fig. 4a, Supplementary Fig. S2 and Supplementary Movie S9), and its moving speed could be adjusted by controlling the voltage exerted on the vibrating motor as shown in Fig. $4 \mathrm{~b}$. And under the same voltage, its moving speed in the tube is much smaller than that of the VDV on the horizontal track. This phenomenon can be interpreted as follows. In one period of vibration, due to the sticking of slant pillars on the jagged surface, the
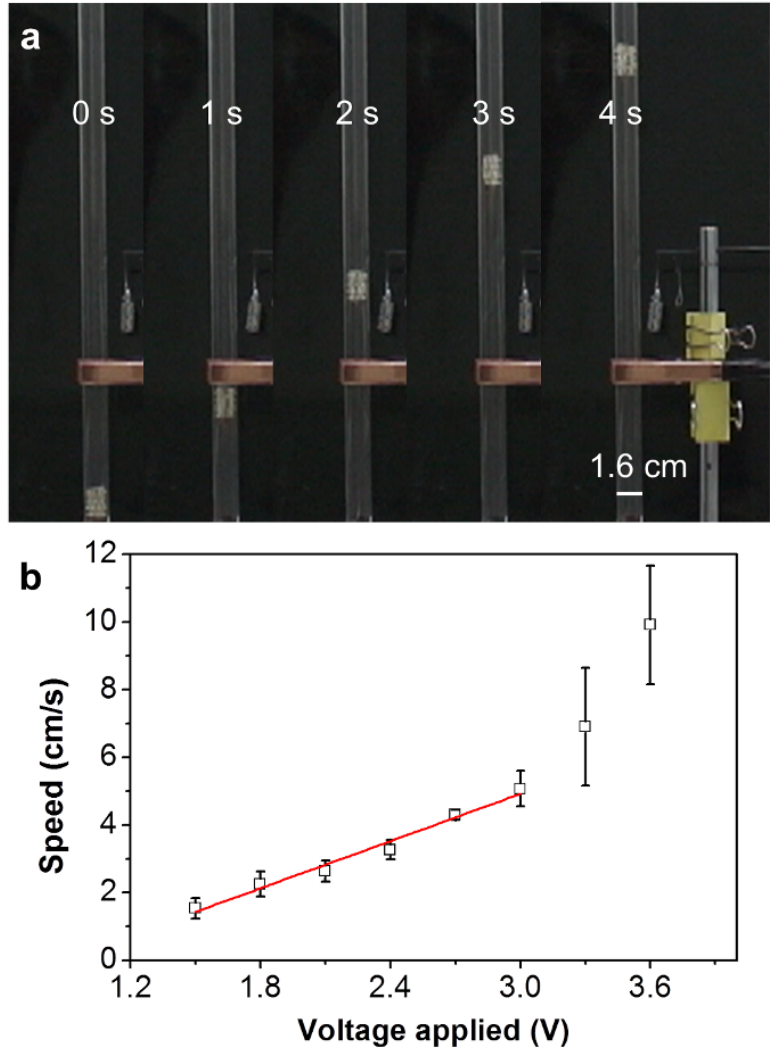

Figure 4 |VDV's vertical motion. (a) Images of a VDV climbing in a vertical glass tube. (b) The relationship between the VDV's speed and the voltage applied on the motor. Below the rated voltage $(3 \mathrm{~V})$ of the motor, a linear fit can be used to represent this relationship. The error bars indicate the standard deviations.

VDV wouldn't fall in the first process, and with a nonzero slant angle $\beta$, the $l$ of VDV has a smaller value compared with that in the horizontal case. According to the equation $v=f_{*} l$, the speed would decrease with the slant angle $\beta$. Finally, using our former reported $\operatorname{method}^{23}$, the patterned film with slant pillar arrays could be reduced to micro/nano-scale, so the VDV could be reduced to micro/nanoscale accordingly, in principle. And in this case, we can use a external stimuli to propel it, which has been proven viable through our preliminary experiment (Supplementary Movie S10). This makes the VDV have the potential applications in nanotechnology such as MEMS, target-drug delivery, in-vivo thrombus removal, et al.

\section{Discussion}

Inspired by the interesting moving behavior of the Setaria viridis spike on a vibrating cotton track, we fabricate a VDV made of PDMS, which makes directional motion utilizing vibrating energy. This new kind of vehicle has demonstrated the following advantages. First, the VDV can work properly in some harsh environments such as industrial pipes, building ruins, which is often encountered in industrial inspection and search-rescue tasks. Second, the VDV demonstrates its outstanding climbing capability in the tilted and even vertical smooth surface of glass, plastic and so on. This makes it have potential use in inspecting the hulls of spacecraft for damage, military reconnaissance, and so on. Third, both the structure and fabricating procedure of our VDV are very simple, which makes our VDV own a high reliability and more suitable for practical applications. Finally, the VDV could be reduced into a micro/nano-scale, which makes VDV has the potential applications in MEMS and biomedical field such as drug delivery. 


\section{Methods}

Grass spike motion measurement. The spike in use is the Setaria viridis spike. Horizontal motion measurement. First, wind a cotton wire around two vertical sticks separated by $48 \mathrm{~cm}$ horizontally to form a track. Second, place the chosen spike on the track and tap one stick with finger continuously. Slant motion measurement. First, attach a $0.21 \mathrm{~g}$ weight via a pin to the spike as a load. Then, wind a cotton wire around two sticks separated by $40 \mathrm{~cm}$ slantwise. The slant angle of the cotton wire is $24^{\circ}$. Finally, place the spike on the track and tap one stick continuously. Vertical motion measurement. First, place a spike in a plastic tube with inner diameter $0.7 \mathrm{~cm}$. Then, tap one end of the plastic tube using finger continuously.

VDV preparation. First, fabricate evenly distributed holes $(1 \mathrm{~mm}$ in length, $1 \mathrm{~mm}$ in width, $5 \mathrm{~mm}$ in depth) on the wax as shown in the left panel of Fig. S3a, which was used as a template. The slant angle of the hole is $45^{\circ}$, and the average spacing between the holes is $2.5 \mathrm{~mm}$. Then fill the holes with PDMS, solidify for one week at the room temperature as shown in the middle panel of Fig. S3a, and then get rid of the wax template. After that, a piece of film with slant pillar arrays is obtained as shown in the right panel of Fig. S3a and Fig. S3b. Roll the film into a tube and use a rubber band to fix it as shown in Fig. 3c. In order to improve the VDV's autonomy for motion control, we place a vibrating alert motor (used for mobile phone's vibrating alerting, rated voltage is $3 \mathrm{~V}$ and rated speed is about $6000 \mathrm{RPM}$ ) into it.

VDV motion measurement. Horizontal motion measurement. First, place the VDV in a straight groove whose inner diameter is $1.8 \mathrm{~cm}$. Then, place the VDV into a groove with different voltages applied to the internal motor. Circular motion measurement. Place the VDV in a horizontal placed circular pipe with $3.2 \mathrm{~V}$ voltage applied to the internal motor. Vertical motion measurement. Place the VDV into a vertical glass tube whose inner diameter is $1.4 \mathrm{~cm}$ with different voltages applied to the internal motor. All VDV's tests are performed on an optical table with the space of $2.54 \mathrm{~cm}$ between the table's neighbor holes.

1. Casper, J. \& Murphy, R. R. Human-robot interactions during the robot-assisted urban search and rescue response at the World Trade Center. IEEE Trans. Syst. Man Cybern. Part B-Cybern. 33, 367-385 (2003).

2. Murphy, R. R. Trial by fire - Activities of the rescue robots at the World Trade Center from 11-21 September 2001. IEEE Robot. Autom. Mag. 11, 50-61 (2004).

3. Murphy, R. R. Marsupial and shape-shifting robots for urban search and rescue. IEEE Intell. Syst. App. 15, 14-19 (2000).

4. Mobedi, B. \& Nejat, G. 3-D Active Sensing in Time-Critical Urban Search and Rescue Missions. IEEE-ASME Trans. Mechatron. 17, 1111-1119 (2012).

5. Granosik, G., Hansen, M. G. \& Borenstein, J. The OmniTread serpentine robot for industrial inspection and surveillance. Ind. Robot 32, 139-148 (2005).

6. Ko, A. W. Y. \& Lau, H. Y. K. Intelligent Robot-assisted Humanitarian Search and Rescue System. Int. J. Adv. Robot. Syst. 6, 121-128 (2009).

7. Erkmen, I., Erkmen, A. M., Matsuno, F. T., Chatterjee, R. \& Kamegawa, T. Snake robots to the rescue! IEEE Robot. Autom. Mag. 9, 17-25 (2002).

8. Bayraktaroglu, Z. Y. et al. in 2006 1st IEEE RAS-EMBS International Conference on Biomedical Robotics and Biomechatronics. 1-3, 277-282.

9. Spenko, M. J. et al. Biologically inspired climbing with a hexapedal robot. J. Field Robot. 25, 223-242 (2008)

10. Murphy, M. P., Tso, W., Tanzini, M. \& Sitti, M. IEEE. in 2006 IEEE/RSJ International Conference on Intelligent Robots and Systems. 1-12, 3411-3416
11. Norman, D. A., Mimlitch, R. H., Galletti, D. M. \& Carter, J. R. Vibration Powered Toy. US patent 8038503 (2011).

12. Barthlott, W. \& Neinhuis, C. Purity of the sacred lotus, or escape from contamination in biological surfaces. Planta 202, 1-8 (1997).

13. Marmur, A. The lotus effect: superhydrophobicity and metastability. Langmuir 20, 3517-3519 (2004).

14. Wilson, S. J. \& Hutley, M. C. The Optical Properties of 'Moth Eye' Antireflection Surfaces. Optica Acta 29, 993-1009 (1982).

15. Gao, X. \& Jiang, L. Biophysics: water-repellent legs of water striders. Nature 432, 36-36 (2004)

16. Ruibal, R. \& Ernst, V. The structure of the digital setae of lizards. J. Morphol. 117, 271-293 (1965).

17. Autumn, K. et al. Adhesive force of a single gecko foot-hair. Nature 405, 681-685 (2000).

18. Blossey, R. Self-cleaning surfaces - virtual realities. Nat. Mater. 2, 301-306 (2003).

19. Huang, Y. F. et al. Improved broadband and quasi-omnidirectional anti-reflection properties with biomimetic silicon nanostructures. Nat. Nanotechnol. 2, 770-774 (2007).

20. Sun, C. H., Jiang, P. \& Jiang, B. Broadband moth-eye antireflection coatings on silicon. Appl. Phys. Lett. 92, 061112 (2008).

21. Kim, T., Jeong, H. E., Suh, K. Y. \& Lee, H. H. Stooped Nanohairs: GeometryControllable, Unidirectional, Reversible, and Robust Gecko-like Dry Adhesive. Adv. Mater. 21, 2276-2281 (2009).

22. Yu, J. et al. Gecko-Inspired Dry Adhesive for Robotic Applications. Adv. Funct. Mater. 21, 3010-3018 (2011).

23. Wu, W. W., Cheng, L., Bai, S., Wang, Z. L. \& Qin, Y. Directional Transport of Polymer Sheet and a Microsphere by a Rationally Aligned Nanowire Array. $A d v$. Mater. 24, 817-821 (2012).

\section{Acknowledgments}

We gratefully acknowledge the financial support from, Fok Ying Tung education foundation (131044), PCSIRT (Grant No. IRT1251), the Fundamental Research Funds for the Central Universities (No. lzujbky-2013-k04).

\section{Author contributions}

Y.Q. and S.B. designed the experiments; S.B. carried out the experiments; all authors analyzed the results; Q.X. and Y.Q. wrote the manuscript.

\section{Additional information}

Supplementary information accompanies this paper at http://www.nature.com/ scientificreports

Competing financial interests: The authors declare no competing financial interests. License: This work is licensed under a Creative Commons Attribution-NonCommercial-NoDerivs 3.0 Unported License. To view a copy of this license, visit http://creativecommons.org/licenses/by-nc-nd/3.0/

How to cite this article: Bai, S., Xu, Q. \& Qin, Y. Vibration driven vehicle inspired from grass spike. Sci. Rep. 3, 1851; DOI:10.1038/srep01851 (2013). 